\title{
El aniversario 140 de la Revista Médica de Chile
}

\author{
HUMBERTO REYES B. ${ }^{1}$, MAX ANDRESEN H. ${ }^{2}$, JOAQUÍN PALMA H. ${ }^{2}$
}

\section{The 140th Anniversary of Revista Médica de Chile}

Revista Médica de Chile was founded in 1872 and thus is one of the oldest medical journals being published since the $19^{\text {th }}$ Century. The sponsoring institution - "Sociedad Médica de Santiago", founded in 1869-initially was the only scientific society in Chile, gathering medical doctors from every existing specialty. With the splitting of independent organizations representing specific specialties, including subspecialties of internal medicine, Sociedad Médica de Santiago focused its scope of action to become the "Chilean Society of Internal Medicine". Its official journal -Revista Médica de Chile- is currently a general and internal medicine journal that also publishes articles on scientific and technological advances in many fields of medicine and health sciences. While initially all authors were Chilean, the journal is now open to submissions from abroad and since the year 2000 articles are published in English when the local language of authors is not Spanish. The number of articles received determines an increasing administrative and editorial burden and, together with the high cost of publishing, will require changes in publication policies. The journal will participate in continuing medical education programs as soon as reaccreditation of medical specialties becomes officially organized in Chile.

(Rev Med Chile 2012; 140: 7-9).

Key words: Education, medical; History of Medicine; Journalism, medical.

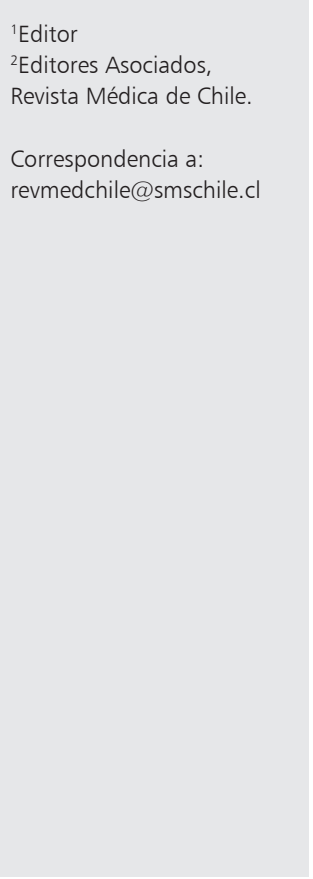

E 1 primer número de la Revista Médica de Chile se imprimió en julio de 1872, como publicación oficial de la Sociedad Médica de Santiago, con el propósito de difundir a los médicos de Chile los avances científicos y clínicos de su profesión. Cuando un aniversario tiene un número relevante (en este caso, 140 parece más interesante que 139 o 141), puede constituirse en efeméride y la celebración se extiende a un año de publicación de la Revista, durante el cual se difunden artículos especiales, que contribuyan al progreso institucional. Las efemérides, o aniversarios simbólicos, invitan a resaltar la trascendencia para la cultura nacional de tener una de las revistas más antiguas que se publican en el mundo ${ }^{1-3}$.

La antigüedad de la Revista Médica de Chile es un fenómeno inusual, ya que en muchos paí- ses -incluso en el hemisferio norte- la aparición y desaparición de las revistas médicas hacen que solo veinticinco de aquellas que se fundaron en el siglo XIX sigan siendo publicadas hoy, y muy pocas conservan su nombre original y el idioma nacional (ver Tabla 1 en la referencia 3$)^{3,4}$.

Chile es un país cuya vida independiente se forjó a comienzos del siglo XIX. Durante ese siglo se fundaron cuatro instituciones relacionadas con medicina, salud pública y educación médica, que continúan vigentes hasta hoy: la Escuela de Medicina (en 1833), ligada inicialmente al Instituto Nacional e incorporada a la Universidad de Chile cuando ésta se fundó (1842), la Sociedad Médica de Santiago (en 1869) y la Revista Médica de Chile (en 1872).

Una razón fundamental para sustentar la vida 
prolongada y fructífera de la Revista Médica de Chile reside en el patrocinio de su entidad propietaria. La Sociedad Médica de Santiago se fundó como una institución científica-profesional sin fines de lucro que, en su inicio, agrupó a médicos generales, cirujanos, obstetras, oftalmólogos, otorrinolaringólogos, patólogos, y a todas las especialidades que fueron naciendo, en concordancia con el progreso de la medicina. La evolución natural condujo a la independencia de las especialidades, incluso de las especialidades derivadas de la medicina interna, con formación de las respectivas sociedades científico-profesionales. Muchas de ellas fundaron sus propias revistas, algunas de las cuales superan ya medio siglo de existencia. La Sociedad Médica de Santiago conservó su nombre histórico y los propósitos generales que le dieron origen, dedicada a la Medicina Interna y General, para todo el país y con sólidas relaciones internacionales. Mientras la Sociedad ha circunscrito sus intereses de acción, su Revista mantiene el rol fundacional de ser una expositora amplia de la evolución de la medicina clínica, la salud pública y la educación médica, en Chile.

Toda revista necesita que se fije un ámbito para los temas que publica. Respetarlo es una función de sus editores, quienes deben tratar de balancear -por una parte- su percepción del interés de los lectores y -por la otra- su responsabilidad de difundir información sobre progresos científicos, técnicos y humanísticos que pueden contribuir a incrementar la cultura médica nacional. En el caso de esta Revista, es conveniente que aborde problemas en que, eventualmente, podrían asumir un rol los médicos internistas o generales de adultos, aunque sean investigaciones realizadas por profesionales no médicos (ejs: psicólogos, sociólogos), o por médicos de otras especialidades y en pacientes ajenos a la medicina interna. También es relevante que difunda temas médicos controversiales, que se debaten en sociedades científicas o a nivel público general, con connotaciones éticas, políticas o religiosas. Así es como cualquier erudito que desee escribir sobre la historia de la medicina, la salud pública y la educación médica en Chile, debe recurrir a ella como una fuente de información ineludible. Sin embargo, la necesidad de fijar un límite a la amplitud de intereses de una revista está determinada porque no solo debe servir al requerimiento de los autores que la solicitan como vía de difusión de sus manuscritos, sino también debe considerarse el interés de los lectores que la utilizan como fuente de conocimientos.

En el año 2007, en una encuesta realizada a los lectores de la Revista Médica de Chile, 63\% de los encuestados declararon dedicar su actividad profesional preferentemente a la medicina interna y $13 \%$ eran médicos generales. En el otro extremo, $14 \%$ de los encuestados se dedicaban a especialidades independientes de la medicina interna o la medicina general de adultos (cirugía, anatomía patológica, neurología, pediatría, genética, urología, dermatología, ginecología, microbiología, radioterapia, toxicología, traumatología, psiquiatría, biología molecular, salud pública, administración en salud) pero son lectores de la Revista porque les interesa su contenido ${ }^{5}$.

La evolución histórica de las revistas provoca situaciones que exigen una búsqueda de soluciones razonables, con la implementación de nuevas políticas. Refrendando la información dada en editoriales previas, el número de manuscritos recibidos anualmente en la Revista ha crecido progresivamente en la década reciente, superando 500 manuscritos en el año 2010. El flujo de manuscritos recibidos del extranjero ha crecido porque desde el año 2000 se acepta publicar en idioma inglés los manuscritos que provienen de países en que el idioma nacional no es el español ${ }^{6,7}$. Ello implica una mayor carga de trabajo para el personal de secretaría y los editores, ocupa horas/ persona, consume material de escritorio y computacional, aumenta el uso de la línea telefónica y del correo electrónico, todo lo cual recarga el costo de producción de la Revista ${ }^{7}$. Por ello, se ha estimado oportuno estudiar con el Directorio de la Sociedad Médica de Santiago una medida que pretende financiar parcialmente el proceso administrativo propio de la recepción de manuscritos.

Otras iniciativas tienen que ver con la programación de nuevas Secciones temáticas, que den beneficios adicionales a sus usuarios. Una de ellas se relaciona con dar facilidades para acceder a programas de educación médica continua, que ayuden a los profesionales que deberán enfrentar una re-acreditación de sus títulos de especialistas en medicina interna y en especialidades derivadas. Pero esta iniciativa requiere que, primero, se oficialice en nuestro país dicha re-acreditación, se redacten y difundan sus reglamentos, y se determinen las entidades responsables de realizarla. Solo cuando se cuente con ese marco, se podrán ofrecer 
programas para educación médica continua que beneficien a quienes deseen tomarlos, porque estarán acreditados legalmente. Pensamos que la Sociedad Médica de Santiago-Sociedad Chilena de Medicina Interna debería tener una participación activa en la organización de un sistema que requiere la preocupación de todas las especialidades de la medicina comprometidas en este proceso.

Mientas se discuten estas ideas y se proporcionan los recursos requeridos para ponerlas en práctica, la Revista Médica de Chile, con sus editores, personal de secretaría y auxiliar, en conjunto con los autores de manuscritos, los especialistas consultados en el proceso de revisión por pares externos y los lectores a quienes está destinada, e interpretando el interés y apoyo del Directorio de la Sociedad Médica de Santiago-Sociedad Chilena de Medicina Interna, inició con entusiasmo la conmemoración de su aniversario número 140, asentado históricamente en una Tradición respetable y respetada y en un ideal de Progreso, para bien de la medicina chilena y como aporte a la ciencia médica universal ${ }^{8,9}$.

\section{Referencias}

1. Reyes H, Kauffmann R, Andresen M. Ciento veinticinco años de publicación continua (Editorial). Rev Med Chile 1997; 125: 133-4.

2. Reyes H. La Revista Médica de Chile cumple 125 años (Editorial). Rev Med Chile 1997; 125: 758-60.

3. Reyes H. El aniversario 135 de la Revista Médica de Chile (Editorial). Rev Med Chile 2007; 135: 7-10.

4. Lee RV. Medical journals: Old, not senescents. A tribute to Revista Médica de Chile on its $135^{\text {th }}$ Anniversary (Editorial). Rev Med Chile 2007; 135: 823-8.

5. Bunout D, Reyes H. ¿Qué opinan sus lectores de la Revista Médica de Chile? Rev Med Chile 2008; 136: 1163-8.

6. Reyes H, Kauffmann R, Andresen M. Tradición y progreso: ¿Ahora también una revista bilingüe? (Editorial). Rev Med Chile 2000; 128: 1187-9.

7. Reyes H, Andresen M, Palma J. La Revista Médica de Chile en el año 2009. Rev Med Chile 2009; 137: 1089-94.

8. Goic A, Reyes H. Tradición y Progreso (Editorial). Rev Med Chile 1973: 101: 111-5.

9. Goic A, Reyes H. De 1872 a 1993: Tradición y Progreso (Editorial). Rev Med Chile 1993: 121: 607-8. 